\title{
Oral v. pulse intravenous cyclophosphamide: A retrospective analysis of adverse events in a setting with a high burden of infectious disease
}

\author{
E Pretorius, ${ }^{1} \mathrm{MB}$ ChB, MMed (Int) FCP (SA); M R Davids, ${ }^{2}$ MB ChB, FCP (SA), MMed (Int); \\ R du Toit, ${ }^{3} \mathrm{MB}$ ChB, MMed (Int), Cert Rheum Phys (SA) \\ ${ }^{1}$ Department of Medicine, Faculty of Medicine and Health Sciences, Stellenbosch University and Tygerberg Academic Hospital, \\ Tygerberg, Cape Town, South Africa \\ ${ }^{2}$ Division of Nephrology, Faculty of Medicine and Health Sciences, Department of Medicine, Stellenbosch University and \\ Tygerberg Academic Hospital, Tygerberg, Cape Town, South Africa \\ ${ }^{3}$ Division of Rheumatology, Department of Medicine, Faculty of Medicine and Health Sciences, Stellenbosch University and \\ Tygerberg Academic Hospital, Tygerberg, Cape Town, South Africa
}

Corresponding author: $R$ du Toit (rdutoit@sun.ac.za)

\begin{abstract}
Background. Cyclophosphamide (CPM) is still considered to be the first-line treatment for many life-threatening autoimmune conditions. It does, however, carry a significant risk of serious adverse events, especially infections. At present CPM is administered as either a daily oral dose (DOC) or an intravenous pulse (PIVC). There is uncertainty regarding the safety profiles of both regimens in settings with a high burden of infectious diseases. Objective. To compare the frequency and nature of adverse events related to the use of DOC and PIVC in such a setting.

Methods. A cohort of patients treated with CPM for autoimmune diseases at Tygerberg Academic Hospital, Cape Town, South Africa, from 1 January 2008 to 31 May 2013 was studied. We compared participants receiving DOC and PIVC with regard to disease characteristics and the occurrence of major adverse events.

Results. A total of 134 participants (92 DOC and 42 PIVC) were included. Participants in the DOC group were treated for longer (174 v. 101 days; $p<0.01$ ) and with higher cumulative doses (17 276 v. $3327 \mathrm{mg} ; p<0.01)$. Risk of infection was similar in the two groups, although there were 6 deaths from leucopenic sepsis in the DOC group (v. $0 ; p=0.18$ ). Nadir leucocyte counts were also lower in the DOC group (median 3.8 v. $5.3 \times 10^{9} / \mathrm{L} ; p=0.02$ ).

Conclusion. Infection rates in the two groups were similar, but DOC was associated with longer treatment duration, greater cumulative $\mathrm{CPM}$ doses and more severe leucopenia. If resources allow and available literature provides support for efficacy, consideration should be given to greater use of PIVC.
\end{abstract}

S Afr Med J 2015;105(3):209-214. DOI:10.7196/SAMJ.8785

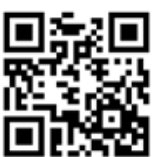

Autoimmune diseases affecting major organs carry significant morbidity and mortality. Immunosuppressive therapy with agents such as cyclophosphamide (CPM) significantly improves quality of life and survival in many patients suffering from such diseases. ${ }^{[1,2]}$

CPM is still considered to be the first-line treatment for many life-threatening autoimmune conditions owing to its well-established therapeutic benefits. It does, however, carry a significant risk of severe adverse events including cytopenias, infections, infertility and bladder toxicity. At present CPM is administered either as a daily oral dose (DOC) or as an intravenous pulse at various intervals (PIVC). The optimal dosing route and regimen remain controversial, especially in the treatment of lupus nephritis.

Large head-to-head trials comparing DOC with PIVC are lacking. Many opinions exist on the optimal dosing regimen, based on small studies or indirect conclusions from larger studies. Overall it seems that DOC has the advantages of lower cost, simpler administration and avoidance of high-dose exposure should the drug be inappropriately administered. ${ }^{[3,4]}$ PIVC has the advantage of a reduced incidence of short- and long-term adverse events and avoids the need for daily compliance with treatment. Data regarding the comparative efficacy of the two regimens are conflicting and briefly discussed below.
Total drug costs for treatment regimens are lower for DOC than for PIVC. PIVC also requires allocated space, consumables and personnel to administer the infusion. Patients need to take time off work and have additional travel expenses, which all contribute to the overall cost of this regimen. ${ }^{[3,4]}$

Although DOC is associated with an increase in long-term sideeffects, adverse events due to inappropriate dosing are more easily managed. Examples include administration when a contraindication exists, mistaking sepsis for a flare of disease or not adjusting doses for renal function. Reducing or omitting further oral doses under these circumstances, which cannot be done with PIVC, reduces the toxicity. ${ }^{[4]}$

Austin et al ${ }^{[5]}$ initially published data in 1986 supporting superior treatment outcomes and reduced side-effect profiles with the use of PIVC as opposed to DOC in patients with lupus nephritis. This led to PIVC becoming the standard treatment in lupus nephritis, with fewer subsequent trials and recommendations that include DOC. ${ }^{[3,6]}$ This practice is now contested, as subsequent trials $s^{[4,7,8]}$ and a recent Cochrane review ${ }^{[9]}$ showed no difference in outcomes or adverse events. An increased tendency to relapse has also been shown in patients with lupus nephritis treated with PIVC. ${ }^{[10]}$ DOC was associated with a higher frequency of adverse events in the same study. Another small trial showed a slightly larger increase in serum 
albumin in patients with membranous nephropathy treated with PIVC in comparison with DOC. ${ }^{[1]}$

Trials in antineutrophil cytoplasmic antibody (ANCA)-associated vasculitides have also suggested equal to increased ability of PIVC to induce remission compared with DOC, but a greater tendency towards relapse. ${ }^{[12-14]}$ Episodes of leucopenia and infection were less frequent in ANCA-associated vasculitides treated with PIVC. ${ }^{[14-17]}$ Increased gonadal toxicity with oral CPM has also been shown in patients treated for ANCA-associated vasculitides. ${ }^{[18]}$ Decreased toxicity with PIVC is thought to be due to the lower cumulative CPM doses used.

No difference in outcomes has been shown between the two regimens in treating pulmonary involvement in scleroderma. ${ }^{[19]}$

The use of CPM in a resource-constrained and infectious diseasesburdened environment, as in our setting at Tygerberg Academic Hospital (TBAH), Cape Town, South Africa, has unique challenges and considerations in respect of cost, efficacy and safety. Cost of treatment to hospitals, lack of allocated space and trained personnel to administer intravenous medication, patients' ability to travel to a tertiary setting to receive treatment, compliance with treatment and follow-up, access to healthcare in the event of drug toxicity, and the overall increased burden of infectious diseases associated with a low socioeconomic environment are all factors to consider in choosing an appropriate treatment plan.

Of special concern in our setting is safety. The high prevalence of tuberculosis (TB) in communities serviced by $\mathrm{TBAH}^{[20]}$ and exposure to overpopulated and unhygienic environments can significantly endanger patients should they become severely immunosuppressed. To our knowledge, no studies comparing DOC and PIVC are available that address these safety issues when CPM is used in such settings.

Clinicians at TBAH make use of both intravenous pulse and oral regimens of CPM for the treatment of autoimmune diseases. Treatment choice is largely based on the condition treated and the preference of the discipline involved in initiating treatment (e.g. lupus nephritis is generally treated with DOC by nephrologists, while vasculitis and other severe systemic complications of connective tissue diseases are treated with PIVC by rheumatologists). As a general guideline, DOC is initiated at a dose of $1.5 \mathrm{mg} / \mathrm{kg}$ and PIVC at $750 \mathrm{mg} / \mathrm{m}^{2}$, and then titrated according to disease severity and leucocyte count. Serious adverse events related to therapy have been encountered with both regimens, but the overall prevalence, differences in frequency and spectrum of adverse events between the two treatment regimens are not known.

\section{Objective}

Our primary objective was to compare the frequency and nature of adverse events related to the use of oral and intravenous CPM in patients with autoimmune diseases.

\section{Methods}

A retrospective cohort study was conducted at TBAH to compare patients treated with DOC with those who were treated with PIVC. Data were extracted from hospital records of adult patients $(>18$ years) suffering from autoimmune diseases treated with CPM. Pharmacy records were used to identify all patients who started treatment between 1 January 2008 and 31 May 2013. Participants were only included if they had been on treatment with CPM for at least 1 month, unless adverse events due to CPM occurred earlier, in which case the participant was also included. Patients who had received CPM for indications other than autoimmune disease (e.g. chemotherapy, transplant recipients) were excluded. Patients on treatment for purely neurological diseases were also excluded owing to the different dosing regimens and treatment duration used.

Data collected included demographics, comorbid disease, mass, diagnosis and disease involvement, duration of disease prior to CPM initiation, treatment prior to $\mathrm{CPM}$ initiation, baseline leucocyte and neutrophil counts, indication for CPM therapy, initial dosage, subsequent adjustment to dosing and indication for changes, cumulative total drug dose, concurrent immunosuppressive drug use, use of preventive therapy (isoniazid, co-trimoxazole, contraception and gonadal protection), documentation of counselling regarding contraception, occurrence of major side-effects and discontinuation due to drug toxicity. Data collected on side-effects included cytopenias, infections, bladder toxicity and infertility. Adverse events were reported and graded according to the Common Terminology Criteria for Adverse Events guidelines. ${ }^{[21]}$

Data were captured on a Microsoft Excel spreadsheet and analysed using Statistica version 11. The two groups were compared with regard to patient characteristics, disease profiles and risk factors for developing major side-effects. Continuous variables were analysed using the Mann-Whitney $U$-test, while categorical data were analysed using the $\chi^{2}$ and Fisher's exact tests. A significance level of 5\% was used throughout.

The study was approved by the Health Research Ethics Committee of Stellenbosch University (protocol No. S13/07/121). Owing to the retrospective nature of the study, the difficulty in tracing individual participants and the low risk to the participants, a waiver of informed consent was granted. Data were collected on standardised capture sheets using study codes assigned to each participant. Codes linking confidential data to the identity of the participants were kept separately and securely.

\section{Results}

After exclusion of oncology patients, 221 subjects were identified. A total of 134 participants were included in the study, 92 in the DOC group and 42 in the PIVC group. Eighty-seven subjects were excluded for reasons that included treatment for non-rheumatological disease, pharmacy errors and missing records, early withdrawal of treatment not related to drug side-effects, failure to follow up after initiation of treatment, concomitant participation in other drug trials, or receiving both PIVC and DOC.

The characteristics of the participants are summarised in Table 1. There were no significant differences between the groups in terms of age, mass, comorbid disease (HIV and diabetes mellitus) and leucocyte count at baseline. Overall, participants were young (mean age 34 years) with a female predominance (74.6\%), especially in the PIVC group ( 85.7 v. $69.6 \%$; $p=0.03$ ).

As expected, there was a marked difference in disease spectrum between the two groups, both in terms of primary diagnosis and organ involvement.

The indications for starting CPM varied significantly. The most frequent indication in the DOC group was class IV lupus nephritis (32.6\%), followed by non-lupus-related renal disease including nephrotic syndrome $(27.1 \%)$ and rapidly progressive (RPGN) or crescentic glomerulonephritis (GN) (21.7\%). The most frequent indication in the PIVC group was neuropsychiatric lupus (45.2\%), followed by interstitial lung disease due to various autoimmune diseases (28.6\%), and lupus myocarditis (28.6\%).

A total of 676 patient-months of treatment were observed. Treatment details are summarised in Table 2. Participants in the PIVC group were treated for significantly shorter periods (mean $101 \mathrm{v} .174$ days; $p<0.01)$ and had much lower mean cumulative doses (3 $327 \mathrm{v}$. $17276 \mathrm{mg} ; p<0.01)$. 


\section{Table 1. Participant baseline characteristics}

\begin{tabular}{|c|c|c|c|c|}
\hline & All $(N=134)$ & DOC $(N=92)$ & $\operatorname{PIVC}(N=42)$ & $p$-value \\
\hline \multicolumn{5}{|l|}{ Gender, $n(\%)$} \\
\hline M & $34(25.4)$ & $28(30.4)$ & $6(14.3)$ & \multirow[t]{2}{*}{0.03} \\
\hline $\mathrm{F}$ & $100(74.6)$ & $64(69.6)$ & $36(85.7)$ & \\
\hline Age (years), mean (SD) & $34.0(12.7)$ & $34.2(13.3)$ & $33.4(11.4)$ & 0.95 \\
\hline Mass (kg), mean (SD) & $69.5(18.4)$ & $70.5(18.0)$ & $67.0(19.5)$ & 0.37 \\
\hline \multicolumn{5}{|l|}{ Comorbid disease, $n(\%)$} \\
\hline Diabetes mellitus & $15(11.2)$ & $9(9.8)$ & $6(14.3)$ & 0.44 \\
\hline HIV & $5(3.7)$ & $5(5.4)$ & $0(0.0)$ & 0.12 \\
\hline \multicolumn{5}{|l|}{ Primary diagnosis, $n(\%)$} \\
\hline SLE & $63(47.0)$ & $41(44.6)$ & $22(52.4)$ & 0.55 \\
\hline Membranous GN & $16(11.9)$ & $16(17.4)$ & $0(0.0)$ & * \\
\hline Mesangiocapillary GN & $7(5.2)$ & $7(7.6)$ & $0(0.0)$ & * \\
\hline SLE/systemic sclerosis overlap & $5(3.7)$ & $3(3.3)$ & $2(4.8)$ & * \\
\hline Crescentic GN & $5(3.7)$ & $5(5.4)$ & $0(0.0)$ & * \\
\hline IgA nephropathy & $5(3.7)$ & $5(5.4)$ & $0(0.0)$ & * \\
\hline Systemic sclerosis & $8(6.0)$ & $2(2.2)$ & $6(14.3)$ & * \\
\hline Granulomatosis with polyangiitis & $11(8.2)$ & $5(5.4)$ & $6(14.3)$ & * \\
\hline FSGS & $2(1.5)$ & $2(2.2)$ & $0(0.0)$ & * \\
\hline Goodpasture's syndrome & $1(0.8)$ & $1(1.1)$ & $0(0.0)$ & * \\
\hline Mesangioproliferative GN & $3(2.2)$ & $3(3.3)$ & $0(0.0)$ & * \\
\hline Minimal-change nephropathy & $2(1.5)$ & $2(2.2)$ & $0(0.0)$ & * \\
\hline Eosinophilic granulomatosis with polyangiitis & $1(0.8)$ & $0(0.0)$ & $1(2.4)$ & * \\
\hline SLE/rheumatoid arthritis overlap & $1(0.8)$ & $0(0.0)$ & $1(2.4)$ & * \\
\hline SLE/dermatomyositis overlap & $1(0.8)$ & $0(0.0)$ & $1(2.4)$ & * \\
\hline SLE/polymyositis overlap & $1(0.8)$ & $0(0.0)$ & $1(2.4)$ & * \\
\hline Rheumatoid arthritis & $1(0.8)$ & $0(0.0)$ & $2(4.8)$ & * \\
\hline Vasculitis of unknown cause & $1(0.8)$ & $0(0.0)$ & $1(2.4)$ & * \\
\hline \multicolumn{5}{|l|}{ Indication for CPM, $n(\%)$} \\
\hline RPGN/crescentic GN other than lupus & $25(18.7)$ & $20(21.7)$ & $5(11.9)$ & 0.18 \\
\hline Nephrotic syndrome other than lupus & $25(18.7)$ & $25(27.2)$ & $0(0.0)$ & $<0.01$ \\
\hline Vasculitis & $4(3.0)$ & $0(0.0)$ & $4(9.5)$ & 0.01 \\
\hline Class III lupus nephritis & $5(3.7)$ & $5(5.4)$ & $0(0.0)$ & 0.12 \\
\hline Class IV lupus nephritis & $31(23.1)$ & $29(31.5)$ & $2(4.8)$ & $<0.01$ \\
\hline Class V lupus nephritis & $7(5.2)$ & $5(5.4)$ & $2(4.8)$ & 0.87 \\
\hline Interstitial lung disease & $20(14.9)$ & $8(8.7)$ & $12(28.6)$ & $<0.01$ \\
\hline Lupus myocarditis & $16(11.9)$ & $4(4.4)$ & $12(28.6)$ & $<0.01$ \\
\hline Eye disease (granulomatosis) & $1(0.8)$ & $1(1.1)$ & $0(0.0)$ & 0.50 \\
\hline Neuropsychiatric lupus & $21(15.7)$ & $2(2.2)$ & $19(45.2)$ & $<0.01$ \\
\hline Diagnosis to initiation of CPM (days), mean (SD) & $23.3(44.8)$ & $17.4(42.2)$ & $36.2(48.1)$ & 0.04 \\
\hline \multicolumn{5}{|c|}{ Duration of disease prior to CPM initiation (months), $n(\%)$} \\
\hline $0-3$ & $70(52.2)$ & $51(55.4)$ & $19(45.2)$ & 0.04 \\
\hline $3-12$ & $19(14.2)$ & $16(17.4)$ & $3(7.1)$ & 0.04 \\
\hline$>12$ & $45(33.6)$ & $25(27.2)$ & $20(47.6)$ & 0.04 \\
\hline Leucocyte count at baseline $\left(\times 10^{9} / \mathrm{L}\right)$, mean $(\mathrm{SD})$ & $9.9(7.7)$ & $9.2(4.3)$ & $11.4(11.9)$ & 0.72 \\
\hline Initiation dose $(\mathrm{mg} / \mathrm{kg})$, mean $(\mathrm{SD})^{\dagger}$ & & $1.6(0.4)$ & $10.3(4.4)$ & \\
\hline
\end{tabular}


Table 2. Treatment characteristics

\begin{tabular}{|c|c|c|c|c|}
\hline & All $(N=134)$ & DOC $(N=92)$ & PIVC $(N=42)$ & $p$-value \\
\hline Treatment duration (days), mean (SD) & $151.4(116.3)$ & $174.0(127.9)$ & $100.9(61.1)$ & $<0.01$ \\
\hline Cumulative dose (mg), mean (SD) & $12903.7(12087.5)$ & 17275.5 (12 210.7) & $3327.4(2489.3)$ & $<0.01$ \\
\hline Cumulative dose (mg/kg), mean (SD) & $193.9(198.0)$ & $262.6(205.5)$ & $46.7(32.5)$ & $<0.01$ \\
\hline \multicolumn{5}{|l|}{ Indication to stop treatment, $n(\%)$} \\
\hline Lost to follow-up & $14(10.5)$ & $9(9.8)$ & $5(11.9)$ & 0.71 \\
\hline Infection & $15(11.1)$ & $10(10.9)$ & $5(11.9)$ & 0.86 \\
\hline Treatment failure & $16(11.9)$ & $15(16.3)$ & $1(2.4)$ & 0.02 \\
\hline Completion of induction & $61(45.5)$ & $36(39.1)$ & $25(59.5)$ & 0.02 \\
\hline Leucopenia & $16(11.9)$ & $13(14.1)$ & $3(7.1)$ & 0.25 \\
\hline Transferred & $1(0.9)$ & $0(0.0)$ & $1(2.4)$ & 0.14 \\
\hline Not stopped & $9(6.7)$ & $8(9.0)$ & $1(2.4)$ & 0.18 \\
\hline
\end{tabular}

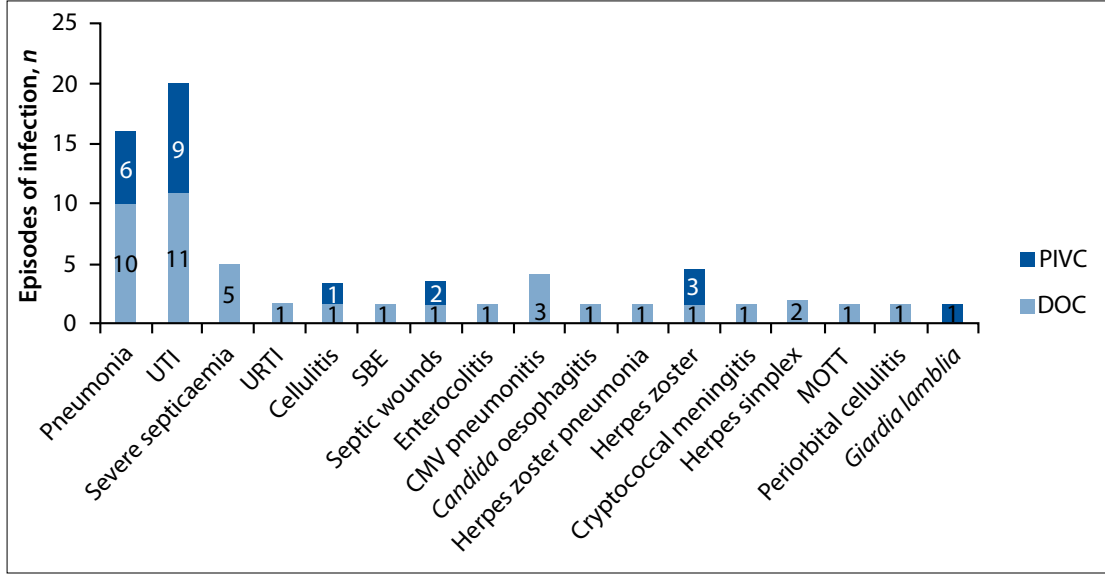

Fig. 1. Infections during CPM treatment. (UTI = urinary tract infection; URTI = upper respiratory tract infection; SBE = subacute bacterial endocarditis; $C M V=$ cytomegalovirus; $M O T T=$ mycobacterium other than TB.)

The most frequent indication for stopping therapy was the successful completion of induction therapy. This was significantly more frequent in the PIVC group, while cessation of treatment due to treatment failure was more frequent in the DOC group (Table 2). Loss to follow-up, infection and leucopenia were also frequent reasons for discontinuation of treatment in both groups. There were nine patients remaining on CPM at the time the study concluded, while one patient had requested to be transferred to a private physician for further management.

Prescription of prophylactic antimicrobials was consistently achieved, with $91.8 \%$ and $94.8 \%$ of all participants receiving isoniazid and co-trimoxazole, respectively. There was no difference between the groups. All patients received concomitant prednisone. Only $43.3 \%$ of the participants who required contraception were documented to have been prescribed contraceptive therapy.

A total of 64 episodes of infection were documented, of which 37 were considered serious and required hospitalisation. The profile of infections is illustrated in Fig. 1. There were no statistically significant differences in the number of infections ( 42 v. $22 ; p=0.53)$ or hospitalisations ( 25 v. 12 ; $p=0.12$ ) between the DOC and PIVC groups. The most frequent infection was urinary tract infection, followed by pneumonia. The most common opportunistic infection was herpes zoster in the PIVC group and CMV pneumonitis in the DOC group. There were no new cases of TB diagnosed in either group. Infections occurred early during therapy (median 57.5 days) in both groups, with no significant difference between the groups (63.0 v. 55.0 days; $p=0.72$ ), but occurred at a higher cumulative dose at the time of infection in the DOC group (7 $964 \mathrm{mg} \mathrm{v}$. $2126 \mathrm{mg} ; p<0.01)$.

Overall, $61.2 \%$ of all participants (59.8\% of the DOC group and $64.3 \%$ of the PIVC group; $p=0.70$ ) had one or more treatment-related adverse events (infection or leucopenia). The relative risk for developing an adverse event in the DOC group v. the PIVC group was 0.93 (95\% confidence interval $0.70-1.23$ ).

Over a third (38.1\%) of participants developed one or more infections during CPM therapy. In the DOC group, $35.9 \%$ of participants developed infections compared with $42.9 \%$ in the PIVC group ( $p=0.45$ ). The overall risk of a participant having one or more hospitalisations as a result of serious infection was $24.6 \%$; this was slightly higher in the DOC group (26.1 v. $21.4 \%$; $p=0.67$ ).

In the DOC group, six participants (6.5\%) died due to leucopenia-related sepsis. There were no treatment-related deaths recorded in the PIVC group. This difference was not statistically significant $(p=0.18)$. Treatmentrelated deaths are summarised in Table 3 .

Because the frequency of monitoring of leucocyte counts was highly variable, we determined the proportion of participants who became leucopenic at any time during therapy, rather than the leucopenia event rate. The lowest recorded leucocyte counts were significantly lower in the DOC group, with a median of 3.8 v. $5.3 \times 10^{9} / \mathrm{L}(p=0.02)$ (Table 4). Leucopenia also occurred later in the DOC group than in the PIVC group (99 v. 76 days; $p=0.04$ ) and at higher cumulative doses (mean 3542 v. 1929 mg; $p<0.01$ ). Notably, neutrophil counts were infrequently done.

Comparing the DOC and PIVC groups, $51.1 \%$ v. $33.3 \% \quad(p=0.06)$ of participants developed leucopenia during treatment. The mean duration of leucopenia before the CPM dose was adjusted or the drug stopped was 5 days, with a maximum delay of up to 28 days. However, delays in stopping CPM appeared not to have contributed to any of the leucopenia-related deaths.

\section{Discussion}

Adverse events due to the use of CPM were frequent in both groups, emphasising 
Table 3. Leucopenia-related deaths

\begin{tabular}{lllll}
\hline $\begin{array}{l}\text { Age (years), } \\
\text { gender }\end{array}$ & Diagnosis & $\begin{array}{l}\text { Duration of } \\
\text { treatment }(\text { days })\end{array}$ & $\begin{array}{l}\text { Lowest leucocyte } \\
\text { count }(\times \mathbf{1 0} / \mathbf{L})\end{array}$ & Cause of death \\
\hline $41, \mathrm{~F}$ & Mesangiocapillary GN with crescents & 59 & 0.79 & Varicella zoster pneumonia \\
$21, \mathrm{~F}$ & SLE with class IV nephritis and myocarditis & 21 & 3.31 & Severe pneumonia and renal failure* \\
$23, \mathrm{~F}$ & SLE with class IV nephritis & 69 & 0.54 & CMV pneumonitis \\
$51, \mathrm{~F}$ & Crescentic GN & 25 & 0.65 & Neutropenic sepsis (unknown source) \\
37, F & SLE with class IV nephritis and myocarditis & 31 & 1.58 & CMV pneumonitis \\
30, F & SLE with class IV nephritis & 49 & 0.34 & Overwhelming Acinetobacter baumannii \\
& & & &
\end{tabular}

the reality that CPM is a potent cytotoxic drug that should be prescribed with caution and with careful follow-up and monitoring. To our knowledge this is the first study from a developing country comparing DOC and PIVC. Other studies have reported leucopenic episodes in $17-26 \%$ of PIVC and $19-45 \%$ of DOC participants ${ }^{[7,14,16]}$ and infection in $10-39 \%$ of PIVC and $14-57 \%$ of DOC participants. Similar infection rates were observed in our study (DOC 36\% v. PIVC $43 \%$ ), while leucopenia was more frequent (DOC 51\% v. PIVC 33\%).

In our setting, it appears that PIVC may be safer than DOC. Six treatment-related deaths occurred in the DOC group and none in the PIVC group, although this difference was not statistically significant. DOC was also associated with more frequent and more severe leucopenia, and with a non-significant increase in hospitalisations suggesting more serious infections, while PIVC was associated with a non-significant increased frequency of all infections.

The participants in the DOC group were treated for a significantly longer time and may therefore have been more likely to experience adverse events. Most adverse events in our study, as in other studies, ${ }^{[16]}$ occurred early in the course of treatment.

It is important to appreciate that in our study the condition being treated determined the route of CPM administration and, by default, also selected for differences in management. Different teams of clinicians and differences in follow-up and monitoring may therefore have affected the results.

The high cumulative doses used in the DOC group could have increased efficacy, but are the likely explanation for the increased risk of leucopenia associated with DOC. Another factor was that PIVC was only administered if there was an acceptable leucocyte count on the day of each pulse infusion. With DOC, a leucocyte count before every dose is not feasible. There was some variability in the frequency of leucocyte counts done in the DOC group. Counts were not always available at the time of follow-up, and were occasionally only reacted to at the next visit. PIVC is perceived as high-dose intravenous chemotherapy and instinctively enforces caution and meticulous follow-up. DOC may be perceived as less potent and not elicit the same careful follow-up by those not familiar with its serious side-effect profile.

This study has identified the need to critically evaluate the current practice of CPM administration at our institution. Most drugattributable deaths and infections occurred early in the course of treatment; it is therefore strongly suggested that leucocyte counts be monitored more frequently soon after initiation of CPM, and that same-day leucocyte counts be available at all follow-up visits. The paucity of neutrophil counts was disappointing. Differential leucocyte counts should be requested routinely, as severe neutropenia may be
Table 4. Lowest recorded leucocyte count in participants who developed leucopenia

\begin{tabular}{llll}
\hline Severity class & $\begin{array}{l}\text { All } \\
\boldsymbol{n}(\%)\end{array}$ & $\begin{array}{l}\text { DOC } \\
\boldsymbol{n}(\%)\end{array}$ & $\begin{array}{l}\text { PIVC } \\
\boldsymbol{n}(\%)\end{array}$ \\
\hline $1(3.01-4.00)$ & $20(32.8)$ & $14(29.8)$ & $6(42.9)$ \\
$2(2.01-3.00)$ & $13(21.3)$ & $8(17.0)$ & $5(35.7)$ \\
$3(1.01-2.00)$ & $13(21.3)$ & $11(23.4)$ & $2(14.3)$ \\
$4(<1.00)$ & $9(14.8)$ & $8(17.0)$ & $1(7.1)$ \\
5 (death) & $6(9.8)$ & $6(12.8)$ & $0(0.0)$ \\
${ }^{*}$ Leucocyte count $\left(\times 10^{9} / \mathrm{L}\right)$ according to Common Terminology Criteria for Adverse Events. ${ }^{[2]}$
\end{tabular}

masked by other cell lines in mild leucopenia if only total leucocyte counts are requested.

An unexpected finding was that no new episodes of TB were documented in any participant. This is especially noteworthy considering the high prevalence of $\mathrm{TB}$ of up to $32 / 1000$ population in communities serviced by this hospital. ${ }^{[20]}$ This suggests that current screening protocols for active TB prior to initiation of CPM and the regular use of isoniazid prophylaxis are effective. Cyclomegalovirus (CMV) pneumonitis was a significant opportunistic infection in the DOC group and a cause of leucopenia-associated death. Patients on CPM who present with leucopenic sepsis should be screened and treated early (and probably empirically) for CMV infection. Current evidence to support prophylactic antiviral treatment for CMV is lacking, and it is unlikely to be cost-effective in a resourceconstrained setting. Consideration could be given to determining patients' CMV status prior to CPM initiation. Knowing the baseline CMV serology and viral load may aid in the decision whether or not to treat for suspected CMV disease when patients on CPM present with leucopenia and signs of infection. However, the costeffectiveness of such an approach would need to be determined.

Alarmingly, almost $10 \%$ of participants initiated on CPM were lost to follow-up immediately after discharge from hospital. Another $10 \%$ were lost to follow-up later in the study. Considering the poor prognosis of the underlying conditions if left untreated, it is possible that many of these patients died as a result of their autoimmune disease. However, adverse events due to CPM are probably a contributing factor. The need for excellent record keeping and tracking of patients on CPM must be emphasised, and clinic staff should be prompted to contact patients should a follow-up visit be missed.

If resources allow, consideration should be given to the greater use of PIVC where its use in the treatment of the specific condition in 
question is supported by current literature. This would be especially useful in cases where compliance with daily medication is in doubt. Owing to the differences in conditions treated, we did not evaluate the efficacy of treatment, which might be the decisive factor in choosing between DOC and PIVC. As pointed out, in many conditions there is a lack of compelling evidence regarding efficacy to preferentially advocate the use of DOC or PIVC.

\section{Study limitations}

This study relied on the completeness and accuracy of TBAH's pharmacy database to identify all patients receiving CPM during the study period. The study was retrospective with data extracted from patient records. A heterogeneous group of conditions were included and allocation to PIVC and DOC was not random but determined by the disease process and the preference of the clinicians involved (rheumatology v. nephrology). The primary disease process as well as differences in practices by clinicians are likely to influence treatment outcomes and risk of adverse events. The long-term complications of CPM (e.g. bladder toxicity, infertility) were not studied.

\section{Conclusion}

This study has provided new data on adverse events due to CPM in a developing world setting with a very high burden of infectious diseases. We found that a greater proportion of participants receiving DOC developed severe leucopenia, which may be explained by the higher cumulative doses taken over longer periods. There was, however, no significant difference in the proportions of participants who developed infections or required hospitalisation as a consequence. Six participants in the DOC group and none in the PIVC group died as a result of complications of CPM treatment; this difference was not statistically significant.

Local randomised trials are needed to compare PIVC and DOC where follow-up is standardised and where homogeneous groups of conditions are compared. This will provide not only more data with regard to safety, but also much-needed data on differences in treatment outcomes.

Acknowledgements. We thank Ms Rhadika Muruvan for help in providing pharmacy data and Dr Justin Harvey from the Stellenbosch University Centre for Statistical Consultation for assistance with data analysis.

\section{References}

. Flossmann O, Berden A, de Groot K, et al. Long-term patient survival in ANCA-associated vasculitis. Ann Rheum Dis 2011;70(3):488-494. [http://dx.doi.org/10.1136/ard.2010.137778]

2. Urowitz MB, Gladman DD, Tom BD, Ibanez D, Farewell VT. Changing patterns in mortality and disease outcomes for patients with systemic lupus erythematosus. J Rheumatol 2008;35(11):2152-2158. [http://dx.doi.org/10.3899/jrheum.080214]

3. Hebert LA, Rovin BH. Oral cyclophosphamide is on the verge of extinction as therapy for severe autoimmune diseases (especially lupus): Should nephrologists care? Nephron Clin Pract 2011;117(1):c8-c14. [http://dx.doi.org/10.1159/000319641]

4. McKinley A, Park E, Spetie D, et al. Oral cyclophosphamide for lupus glomerulonephritis: An McKinley A, Park E, Spetie D, et al. Oral cyclophosphamide for lupus glomerulonephritis: An
underused therapeutic option. Clin J Am Soc Nephrol 2009;4(11):1754-1760. [http://dx.doi. rg/10.2215/CJN.02670409]

5. Austin HA 3rd, Klippel JH, Balow JE, et al. Therapy of lupus nephritis: Controlled trial of prednisone and cytotoxic drugs. N Engl J Med 19866;314(10):614-619. [http://dx.doi.org/10.1056/ EJM198603063141004

6. Bargman JM. How did cyclophosphamide become the drug of choice for lupus nephritis? Nephrol Dial Transplant 2009;24(2):381-384. [http://dx.doi.org/10.1093/ndt/gfn640

7. Yee CS, Gordon C, Dostal C, et al. EULAR randomised controlled trial of pulse cyclophosphamide and methylprednisolone versus continuous cyclophosphamide and prednisolone followed by azathioprine and prednisolone in lupus nephritis. Ann Rheum Dis 2004;63(5):525-529. [http://dx.doi.org/10.1136/ ard.2002.003574]

8. Bansal VK, Beto JA. Treatment of lupus nephritis: A meta-analysis of clinical trials. Am J Kidney Dis 1997;29(2):193-199. [http://dx.doi.org/10.1016/S0272-6386(97)90029-9]

9. Henderson L, Masson P, Craig JC, et al. Treatment for lupus nephritis. Cochrane Database Syst Rev 2012;12:CD002922. [http://dx.doi.org/10.1002/14651858.CD002922.pub3]

10. Mok CC, Ho CT, Siu YP, et al. Treatment of diffuse proliferative lupus glomerulonephritis: A comparison of two cyclophosphamide-containing regimens. Am J Kidney Dis 2001;38(2):256-264.
cons [http://dx.doi.org/10.1053/ajkd.2001.26084]

11. Dede F, Ayili D, Sahiner S. Effective treatment administration of cyclophosphamide in membranous nephropathy. J Nephrol 2008;21(4):560-565.

12. Guillevin L, Cordier JF, Lhote F, et al. A prospective, multicenter, randomized trial comparing steroids and pulse cyclophosphamide versus steroids and oral cyclophosphamide in the treatment of generalized Wegener's granulomatosis. Arthritis Rheum 1997;40(12):2187-2198 [http://dx.doi. org/10.1002/art.1780401213

13. Harper L, Morgan MD, Walsh M, et al. Pulse versus daily oral cyclophosphamide for induction of remission in ANCA-associated vasculitis: Long-term follow-up. Ann Rheum Dis 2012;71(6):955-960. [http://dx.doi.org/10.1136/annrheumdis-2011-200477]

14. De Groot K, Adu D, Savage CO, EUVAS (European Vasculitis Study Group). The value of pulse cyclophosphamide in ANCA-associated vasculitis: Meta-analysis and critical review. Nephrol Dial Transplant 2001;16(10):2018-2027. [http://dx.doi.org/10.1093/ndt/16.10.2018]

15. Haubitz M, Schellong S, Gobel U, et al. Intravenous pulse administration of cyclophosphamide versus daily oral treatment in patients with antineutrophil cytoplasmic antibody-associated vasculitis and daily oral treatment in patients with antineutrophil cytoplasmic antibody-associated vasculitis and
renal involvement: A prospective, randomized study. Arthritis Rheum 1998;41(10):1835-1844 [http:// renal involvement: A prospective, randomized study. Arthritis Rheum 1998;41
dx.doi.org/10.1002/1529-0131(199810)41:10<1835::AID-ART16>3.0.CO;2-Q]

16. De Groot K, Harper L, Jayne DR, et al. Pulse versus daily oral cyclophosphamide for induction of remission in antineutrophil cytoplasmic antibody-associated vasculitis: A randomized trial. Ann Intern Med 2009;150(10):670-680. [http://dx.doi.org/10.7326/0003-4819-150-10-200905190-00004]

17. Gayraud M, Guillevin L, Cohen P, et al. Treatment of good-prognosis polyarteritis nodosa and ChurgStrauss syndrome: Comparison of steroids and oral or pulse cyclophosphamide in 25 patients. French Cooperative Study Group for Vasculitides. Br J Rheumatol 1997;36(12):1290-1297. [http://dx.doi. org/10.1093/rheumatology/36.12.1290]

18. Haubitz M, Ehlerding C, Kamino K, Koch KM, Brunkhorst R. Reduced gonadal toxicity after i.v. cyclophosphamide administration in patients with nonmalignant diseases. Clin Nephrol 1998;49(1):19-23.

19. Davas EM, Peppas C, Maragou M, Alvanou E, Hondros D, Dantis PC. Intravenous cyclophosphamide pulse therapy for the treatment of lung disease associated with scleroderma. Clin Rheumatol 1999;18(6):455-461. [http://dx.doi.org/10.1007/s100670050138]

20. Claassens M, van Schalkwyk C, den Haan L, et al. High prevalence of tuberculosis and insufficient case detection in two communities in the Western Cape, South Africa. PLoS One 2013;8(4):e58689. [http:// dx.doi.org/10.1371/journal.pone.0058689]

21. CTCAE Files. http://evs.nci.nih.gov/ftp1/CTCAE/About.html (accessed 30 July 2014).

Accepted 12 January 2015 